\title{
Supernova Neutrino Oscillations *
}

\author{
H. Nunokawa ${ }^{\mathrm{a}}$ \\ a Instituto de Física Gleb Wataghin, Universidade Estadual de Campinas, UNICAMP \\ 13083-970 Campinas SP, Brazil
}

We discuss some possible influence of neutrino oscillations on physics of supernova mainly focusing on the observations of $\bar{\nu}_{e}$, and present some analysis of SN1987A data in the light of three neutrino mixing scheme.

\section{Introduction}

Sucessful observation of neutrino burst from the supernova SN1987A, which is believed to be the collapse driven one, in the Large Magellanic Cloud by Kamiokande-II [1] and IMB [2] detectors have confirmed the basic picture of supernova explosion (see Refs. [3 4 4 for a review).

Such collapse driven supernova can be a good laboratory to test various unknown neutrino properties not only mass and flavor mixing but also other properties such as magnetic moment, life time and some other non-standard interactions 四.

It has been known that neutrino flavor conversion by the MSW effect [5] inside supernova could cause some significant influence on supernova physics 4,6 8. In this talk we review the possible influence of neutrino flavor conversion on supernova physics and present some analysis of the SN1987A data in light of three flavor mixing scheme of neutrinos, based on our recent work [9].

\section{Standard picture of supernova neutrino emission}

Here we summarize the basic understanding of neutrinos from supernova (SN) [3] and their properties inside neutrinosphere 1012 .

A type-II supernova occurs when a massive star $\left(M \gtrsim 8 M_{\odot}\right)$ has reached the last stage of its life. Almost all $(\sim 99 \%)$ of the gravitational binding energy of the final neutron star (about $\sim 10^{53} \mathrm{erg}$ )

\footnotetext{
*Talk presented by H. Nunokawa at Euresco EuroConference Series on Frontiers in Particle Astrophysics and Cosmology, San Feliu de Guixols, Spain, September 30October 5, 2000.
}

is radiated away in form of all flavors of neutrinos. The individual total neutrino luminosities in supernovae are approximately the same for all flavors but the individual neutrino energy distributions are very different 10 13]. This is because different flavor of neutrinos interact differently with the star material, as implied by the following reactions,

$$
\begin{aligned}
& \nu_{e}+n \rightarrow p+e^{-}, \\
& \bar{\nu}_{e}+p \rightarrow n+e^{+}, \\
& \nu+N \rightarrow \nu+N, \quad(N=p, n) .
\end{aligned}
$$

Since the cross sections of the charged-current reaction is larger than that of the neutral-current one and there are more neutrons than protons, the $\nu_{e}$ 's have the largest interaction rates with the matter and hence thermally decouple at the lowest temperature. On the other hand, $\nu_{\tau(\mu)}$ and $\bar{\nu}_{\tau(\mu)}$ 's lack the the charged-current absorption reactions on the free nucleons inside the neutron star and hence thermally decouple at the highest temperature.

As a result, the average neutrino energies satisfy the following hierarchy:

$$
\left\langle E_{\nu_{e}}\right\rangle\left\langle\langle E _ { \overline { \nu } _ { e } } \rangle \left\langle\left\langle E_{\nu_{\tau(\mu)}}\right\rangle \approx\left\langle E_{\bar{\nu}_{\tau(\mu)}}\right\rangle .\right.\right.
$$

Typically, the average supernova neutrino energies are, $\left\langle E_{\nu_{e}}\right\rangle \approx 11-12 \mathrm{MeV},\left\langle E_{\bar{\nu}_{e}}\right\rangle \approx 14-$ $17 \mathrm{MeV},\left\langle E_{\nu_{\tau(\mu)}}\right\rangle \approx\left\langle E_{\bar{\nu}_{\tau(\mu)}}\right\rangle \approx 24-27 \mathrm{MeV}$. Such energy hierarchy is of crucial importance for our discussion as we will see later.

The shape of the energy spectra of various flavors of neutrinos can be described by a "pinched" Fermi-Dirac distribution 114. The pinched form can be parametrized by introducing an effective 
"chemical potential", $\eta$ as follows,

$F\left(E_{\nu}\right) \propto \frac{E^{2}{ }_{\nu}}{1+\exp \left[-E_{\nu} / T-\eta\right]}$,

where $T$ is the temperature.

There is no physical, significant distinction between $\nu_{\mu}$ and $\nu_{\tau}$ and their antiparticles in neutrinosphere. It is because $\nu_{\mu}$ and $\bar{\nu}_{\mu}$ are not energetic enough to produce muons by the charged current interactions, and the neutral current cross sections of $\nu$ and $\bar{\nu}$ are similar in magnitude. Therefore, following Ref. 9 we collectively denote them as "heavy falvor neutrinos", $\nu_{\text {heavy }}$, in this talk even if they are not heavy (as in the case of nverted mass hierarchy we will discuss later).

\section{Possible influence of neutrino oscillation on supernova physics}

Here we briefly review possible effects of neutrino oscillation on supernova physics, which were discussed in number of previous works.

First let us stress some characteristic features of neutrino oscillations in supernova. Because of extremely high matter density around neutrinosphere, MSW resonant neutrino conversion [5] can occur for the mass squared difference much larger than the ones relevant for solar or atmospheric neutrinos. Because of this, in general, one must deal with multiple resonances in supernova (in contrast to the case of solar neutrinos) in the case of three (or more) flavor mixing scheme.

Another important point is that because supernova emit all flavors of neutrinos, if $\nu_{\alpha} \rightarrow \nu_{\beta}$ or $\bar{\nu}_{\alpha} \rightarrow \bar{\nu}_{\beta}(\alpha, \beta=$ e, heavy $)$ occurs, $\nu_{\beta} \rightarrow \nu_{\alpha}$ or $\bar{\nu}_{\beta} \rightarrow \bar{\nu}_{\alpha}$ also must occur simultaneously, and the net effect in the case of large oscillation probability is the exchange of the energy spectrum of $\nu_{\alpha}$ and $\nu_{\beta}$ or $\bar{\nu}_{\alpha}$ and $\bar{\nu}_{\beta}$.

\subsection{Shock reheating}

Neutrino conversion could have significant impact on shock re-heating in the delayed explosion scenario [15]. If the conversion between $\nu_{e}$ and $\nu_{\text {heavy }}$ occurs in the region between the neutrinosphere and the stalled shock this can help the explosion [7]. Due to the conversion the energy spectra of $\nu_{e}$ and $\nu_{\text {heavy }}$ can be swapped and hence $\nu_{e}$ would have larger average energy leading to a larger energy deposition by reactions in eqs. (11) and (2) so that the stalled shock would be reenergized. In order for this effect to be operative, $\Delta m^{2} \gtrsim 100 \mathrm{eV}^{2}$ is required [7].

\subsection{Heavy elements nucleosynthesis}

In nature, supernova is considered to be one of the most promising site to create neutron rich heavy elements [16]. To have successful $r$-process the site must be neutron rich, i.e. $Y_{e}<0.5$ where $Y_{e}$ is number of electron per baryon. The $Y_{e}$ value is mainly determined by the competition between the two absorption reactions in eqs. (1) and (2). In the standard supernova model the latter process is favoured due to the higher average energy of $\bar{\nu}_{e}$ which guarantees the neutron richness. If the neutrino oscillations in $\nu_{e}-\nu_{\text {heavy }}$ channel do occur between the neutrinosphere and the region relevant for $r$-process the site can be driven to proton-rich due to the reaction (11) and therefore, $r$-process could be prevented [8]. Using this argument, mixing parameters in the region $\Delta m^{2} \gtrsim$ few $\mathrm{eV}^{2}$ and $\sin ^{2} 2 \theta \gtrsim 10^{-5}-10^{-4}$ can be excluded $[8$.

\subsection{Influence for $\nu_{e}$ induced events}

If $\nu_{e}-\nu_{\text {heavy }}$ oscillation occurs, some enhancement of forward peaking elastic scattering events at high energies which should be observable in water Cherenkov detectors [17, as well as enhanced oxygen-induced events due to a steep rise of the cross section at energies higher than $\gtrsim$ $30 \mathrm{MeV} 18$, which could be separated from the dominant isotropic $\overline{\nu_{e}}$ absorption events due to a moderate backward peaking of the events 19.

\section{4. $\bar{\nu}_{e}$ signal}

Last argument is the effect of neutrino oscillation on $\bar{\nu}_{e}$ signal in the terrestrial detector, which will be discussed more in detail in the following sections. If large oscillation between $\bar{\nu}_{e}$ and $\bar{\nu}_{\text {heavy }}$ occurs, the $\bar{\nu}_{e}$ spectrum gets harder and can affect significantly the observation of supernova neutrinos, because the cross section for the $\bar{\nu}_{e} p$ absorbed charged current reaction is much larger than that of the $\nu_{e} e^{-}$elastic one. Applying this argument, vacuum oscillation and large mixing angle MSW solutions to the solar neutrino problem are disfavored by the observed SN1987A 
neutrino data at Kamiokande [1] and IMB [2] detectors [20 22]. We will discuss in sec. 5 that how this conclusion could be modified or to be interpreted in the context of three neutrino mixing scheme.

\section{Neutrino conversion in three flavor scheme}

We now discuss the neutrino flavor conversion in supernova $(\mathrm{SN})$, in the three flavor mixing scheme. To be most conservative, here we assume neutrino mixing only among three active neutrino flavors, i.e., among $\nu_{e}, \nu_{\mu}, \nu_{\tau}$ and among their anti-particles, and do not consider the conversion into sterile neutrinos.

Here, we assume oscillation interpretation of the atmospheric 23] and solar neutrino 24] data and do not consider the LSND result [25]. Some possible implications of the mixing scheme which can explain the LSND result have been discussed in Refs. 26 28.

We note that two $\Delta m^{2}$ which can explain atmospheric as well as solar neutrinos are irrelevant for the shock reheating and heavy elements nucleosynthesis (see secs. 3.1 and 3.2) and therefor, we will discuss the influence only on $\nu_{e}$ and $\bar{\nu}_{e}$ signals, mainly focusing on the latter.

The first very important question is if the neutrino mass spectrum adopts the normal or inverted mass hierarchies, because it determines wheather there is a resonant conversion in the antineutrino channel or not. These two mass hierarchies are illustrated schematically in Fig. 1, in which we assume that smaller mass squared difference is in the range relevant to explain the solar neutrino data and the larger one for atmospheric neutrino data.

In these mass hierarchies, the three neutrino and three antineutrino eigenstates have two level crossings, first at higher $(\mathrm{H})$ density and the second at lower (L) density, inside SN as schematically illustrated in Fig. 2. If the mass hierarchies is of normal (inverted) type, the $\mathrm{H}$ level crossing is in the neutrino (antineutrino) channel.

The second important question is that if the neutrino (or antineutrino) flavor conversion in SN at $\mathrm{H}$ level crossing is adiabatic or not. If it is very

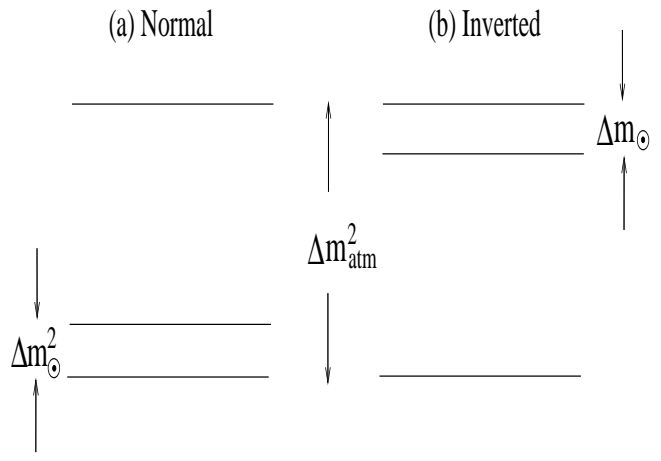

Fig. 1: Mass hierarchy schemes we consider.

adiabatic, then the physical properties of neutrino conversion is simply $\nu_{e}-\nu_{\text {heavy }}$ or $\bar{\nu}_{e}-\bar{\nu}_{\text {heavy }}$ exchange in the case of normal or inverted mass hierarchy. It should be emphasized that this feature holds irrespective of the possible complexity of the solar neutrino conversion which governs the $\mathrm{L}$ resonance. These key features have been pointed out in our earlier paper, Ref. [17] in the case of normal mass hierarchy.

If the mass hierarchy is inverted and $\mathrm{H}$ resonance is adiabatic, then $\bar{\nu}_{e}$ spectrum gets harder. Since the $\bar{\nu}_{e}$-induced charged current reaction is dominant in water Cherenkov detector, one can severely constrain the scenario of inverted mass hierarchy by utilizing this feature of neutrino flavor transformation in SN. When the next supernova event comes it can be used to make clear judgement on whether the inverted mass hierarchy is realized in nature, a completely independent information from those that will be obtained by the future long-baseline neutrino oscillation experiments 29 31], or by the neutrinoless double beta decay experiments [32] for the case of Majorana neutrinos.

The adiabaticity of the $\mathrm{H}$ resonance is guaranteed if the following adiabaticity parameter $\gamma$ is significantly larger than unity at the resonance point:

$$
\begin{aligned}
\gamma & \equiv \frac{\Delta m^{2}}{2 E} \frac{\sin ^{2} 2 \theta}{\cos 2 \theta}\left|\frac{\mathrm{d} \ln N_{e}}{\mathrm{~d} r}\right|_{\text {res }}^{-1} \\
= & \left(\frac{\Delta m^{2}}{2 E}\right)^{1-1 / n} \frac{\sin ^{2} 2 \theta}{(\cos 2 \theta)^{1+1 / n}} \frac{r_{\odot}}{n}\left[\frac{\sqrt{2} G_{F} \rho_{0} Y_{e}}{m_{p}}\right]^{1 / n}(6)
\end{aligned}
$$



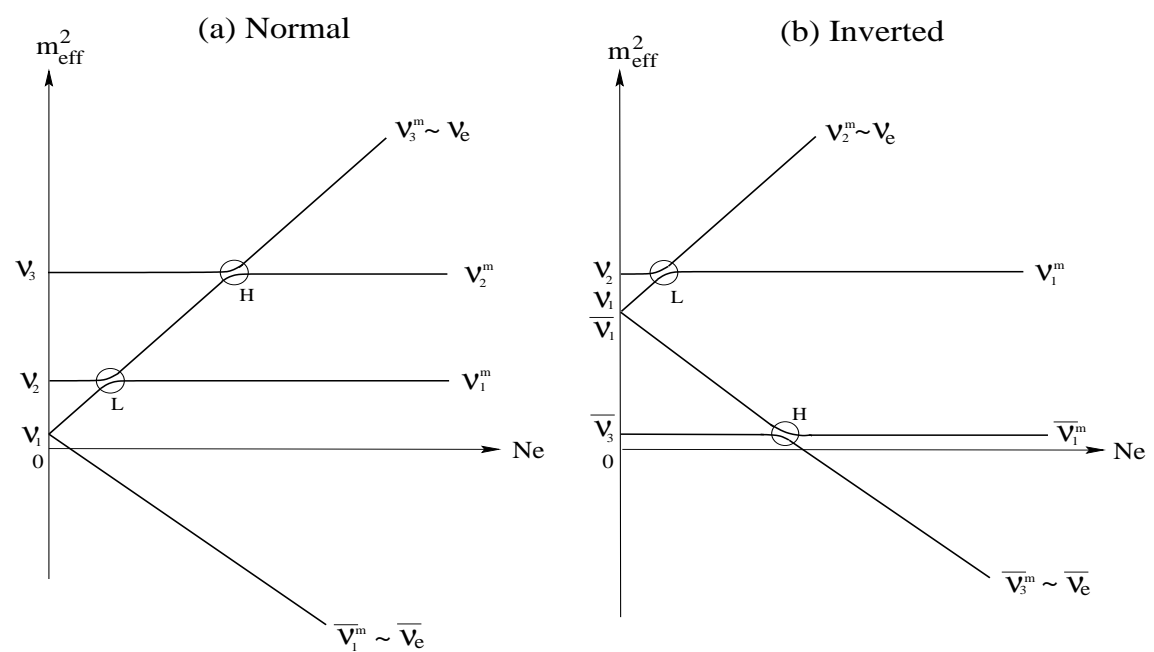

Fig. 2: The schematic level crossing diagram for the case of (a) normal and (b) inverted mass hierarchies considered in this work. The circles with the symbol H and L correspond to resonance which occur at higher and lower density, respectively. Adopted from Ref. 99

Here, we assumed that the density profile of the relevant region of the star can be described as $\rho(r)=\rho_{0}\left(r / r_{\odot}\right)^{-n}$ to obtain the second line in the above equation, where $r_{\odot}=6.96 \times 10^{10} \mathrm{~cm}$ denotes the solar radius. With the choice $n=3$ and $\rho_{0} \simeq 0.1 \mathrm{~g} / \mathrm{cc}[33]$, we get,

$\gamma \simeq 0.63 \times\left[\frac{\sin ^{2} \theta_{13}}{10^{-4}}\right]\left[\frac{\Delta m^{2}}{10^{-3} \mathrm{eV}^{2}}\right]^{2 / 3}\left[\frac{E}{20 \mathrm{MeV}}\right]^{-2 / 3}$,

for the small value of $\theta_{13}$. Since the conversion probability $P$ is approximately given by $P_{H} \simeq$ $\exp \left[-\frac{\pi}{2} \gamma\right], \sin ^{2} \theta_{13} \gtrsim$ a few $\times 10^{-4}$ assures adiabaticity in a good accuracy.

For a recent complehensive treatment of neutrino flavor conversion in $\mathrm{SN}$ in the framework of three-flavor mixing, see Ref. [34].

\section{Analaysis of the supernova SN1987A neutrino data in the light of three neu- trino scheme}

While waiting for the next galactic SN, let us perform an analysis of the data of neutrinos from SN1987A to gain a hint to the problem of the mass and mixing pattern which we want to solve.

We repeat the similar analyses performed in Refs. [20,21 but in the context of three-flavor mixing scheme of neutrinos, which is essential for the SN neutrinos. In due course, we will try to clarify how conclusions obtained in earlier works are to be interpreted, or to be conditioned in the three-flavor framework.

We follow the statistical analysis of the SN1987A data performed by Jegerlehner, Neubig and Raffelt [21] who employed the method of maximum likelihood. Using the same Likelihood function which can be found in Ref. [21], we perform a fit of the observed data to the two parameters, the binding energy of the neutron star, $E_{b}$, and the $\bar{\nu}_{e}$ temperature, $T_{\bar{\nu}_{e}}$.

First we show the result of our analysis without assuming neutrino oscillation. In Fig. 3 we show the contours of constant likelihood for Kamiokande and IMB data, as well as the combined one.

For simplicity, as in Ref. 21], we set the "effective" chemical potential equal to zero in the neutrino distribution functions because we believe that our results would not depend much even if we introduce some non-zero chemical potential. We note that our result is in very good agreement with the one obtained in Ref. [21].

We note that in Fig. 3, the agreement between Kamionde and IMB data is not so significant though these data are consistent. The possibility to reconcile the difference between Kamionde and IMB data by utilizing the earth matter effect 
has been recently considered in Ref. [35]. Here, we do not enter into this point and simply try to do the combined fit as in Ref. [21].

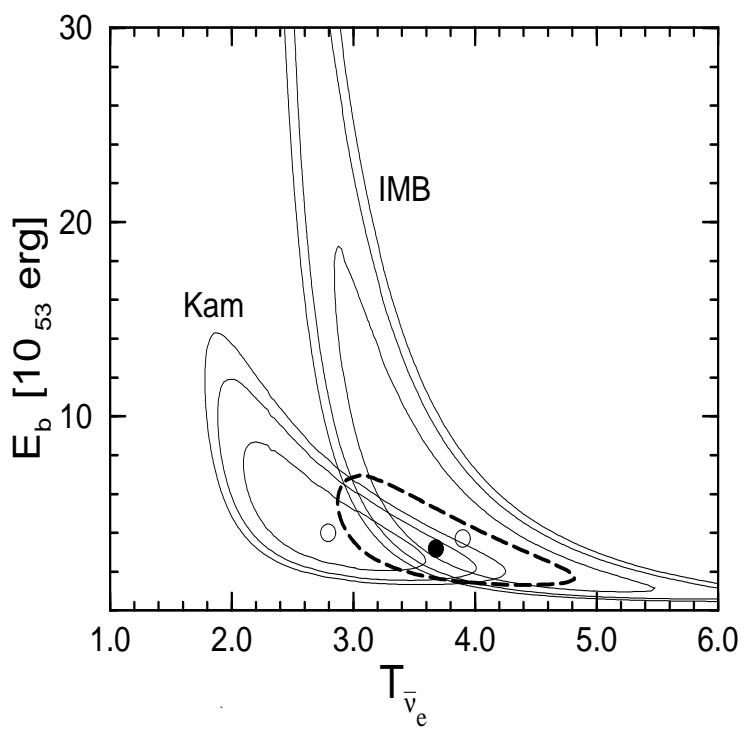

Fig. 3: Contours of constant likelihood corresponding to $68.3,90$ and $95.4 \%$ C.L. region in $E_{b}-T_{\bar{\nu}_{e}}$ for Kamiokande and IMB data. Best fitted points are indicated by open circles. Think dashed curve shows the combined $95.4 \%$ C.L. contour and the solid circle indicates the combined best fit.

\subsection{Case I}

Let us first consider the case where the observable effect is most significant: the mass hierarchy is inverted and $\mathrm{H}$ resonance is very adiabatic, which can be realized if $\sin ^{2} \theta_{13} \gtrsim 10^{-4}$. In this case there is a efficient conversion in $\bar{\nu}$ channel at $\mathrm{H}$ resonance.

We draw in Fig. 4 equal likelihood contours as a function of the heavy to light temperature ratio $\tau$ on the space spanned by $\bar{\nu}_{e}$ temperature and total neutrino luminosity by giving the neutrino events from SN1987A observed by Kamiokande [1] and IMB [2] detectors. We characterize the difference in energy by the temperature ratios of $\nu_{e}$ and $\bar{\nu}_{e}$ to $\nu_{\text {heavy }}$ as

$\tau \equiv \frac{T_{\nu_{\text {heavy }}}}{T_{\bar{\nu}_{e}}} \simeq \frac{T_{\bar{\nu}_{\text {heavy }}}}{T_{\bar{\nu}_{e}}}$.

According to the simulation of supernova dynamics which is carried out in Ref. [10 12], typically $\tau \simeq 1.4-2.0$.

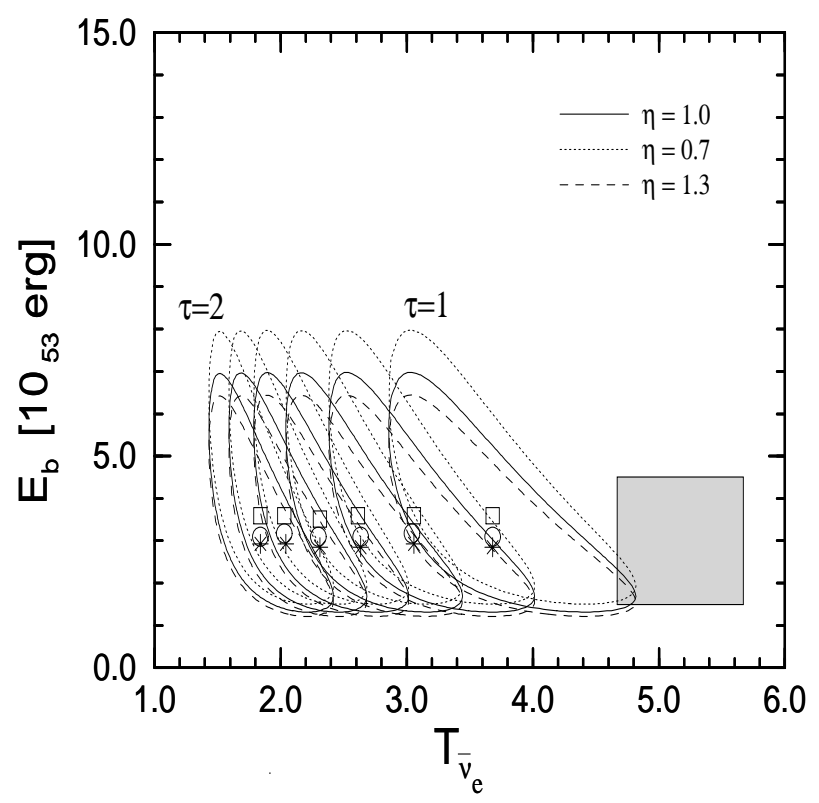

Fig. 4: Contours of constant likelihood which correspond to $95.4 \%$ confidence regions for the inverted mass hierarchy under the assumption of adiabatic $\mathrm{H}$ resonance. From left to right, $\tau \equiv T_{\bar{\nu}_{\text {heavy }}} / T_{\bar{\nu}_{e}}=$ $T_{\nu_{\text {heavy }}} / T_{\bar{\nu}_{e}}=2,1.8,1.6,1.4,1.2$ and 1.0. Best-fit points for $T_{\bar{\nu}_{e}}$ and $E_{b}$ are also shown by the open circles. The parameter $\eta$ parametrizes the departure from the equipartition of energy, $L_{\nu_{\text {heavy }}}=L_{\bar{\nu}_{\text {heavy }}}=$ $\eta L_{\nu_{e}}=\eta L_{\bar{\nu}_{e}}$, and the dotted lines (with best fit indicated by open squares) and the dashed lines (with best fit indicated by stars) are for the cases $\eta=0.7$ and 1.3, respectively. Theoretical predictions from supernova models are indicated by the shadowed box. Adopted from Ref. []

In addition to it we introduce an extra parameter $\eta$ defined by $L_{\nu_{\text {heavy }}}=L_{\bar{\nu}_{\text {heavy }}}=\eta L_{\nu_{e}}=\eta L_{\bar{\nu}_{e}}$ which describe the departure from equipartition of energies to three neutrino species and examine the sensitivity of our conclusion against the change in the SN neutrino spectrum.

At $\tau=1$, that is at equal $\bar{\nu}_{e}$ and $\nu_{e}$ temperatures, the $95 \%$ likelihood contour marginally overlaps with the theoretical expectation [12] represented by the shadowed box in Fig. 4. When the temperature ratio $\tau$ is varied from unity to 2 the likelihood contour moves to the left, indicat- 
ing less and less consistency, as $\tau$ increases, between the standard theoretical expectation and the observed feature of the neutrino events after the MSW effect in $\mathrm{SN}$ is taken into account. This is simply because the observed energy spectrum of $\bar{\nu}_{e}$ must be interpreted as that of the original one of $\bar{\nu}_{\text {heavy }}$, in the presence of the MSW effect in the anti-neutrino channel, which implies that the original $\bar{\nu}_{e}$ temperature must be lower by a factor $\tau$ than the observed one, leading to stronger inconsistency at larger $\tau$.

The solid lines in Fig. 4 are for the case of equipartition of energy into three flavors, $\eta=1$, whereas the dotted and the dashed lines are for $\eta=0.7$ and 1.3 , respectively. We observe that our result is very insensitive against the change in $\eta$.

We conclude that if the temperature ratio $\tau$ is in the range 1.4-2.0 as the SN simulations indicate, the inverted hierarchy of neutrino masses is disfavored by the neutrino data of SN1987A unless the $\mathrm{H}$ resonance is nonadiabatic. We note that similar conclusion is obtained also in Ref. [35].

\subsection{Case II}

Let us next consider the case where mass hierarchy is normal or inverted but $\mathrm{H}$ resonance is very non-adiabatic, which correspond to the case with very small $\theta_{13}, \sin ^{2} \theta_{13} \ll 10^{-4}$. In this case, there is no (or no significant) adiabatic conversion in $\bar{\nu}$ channel, and therefore, as far as $\bar{\nu}_{e}$ signal is concerned, the problem is essentially reduced to that in the two flavor scheme which was studied in Refs. 20.21].

Conclusions can be summarized as follows: If the small mixing angle (SMA) MSW is the solution to the solar neutrino problem there is only a minor effect because neither the vacuum oscillation nor the earth matter effects are effective because of small $\theta_{12}$. If the large mixing angle (LMA) or low $\Delta m^{2}$ (LOW) MSW or vacuum oscillation (VO) is the solution, we have a potential trouble because a good fraction of $\bar{\nu}_{e}$ is transformed into $\bar{\nu}_{\text {heavy }}$ and vice versa.

We have repeated the same analysis as done in Ref. 21] and obtained very similar results. We conclude that VO and LOW solutions are more disfavored than the LMA MSW solution mainly because of the absence of earth matter effect for the former solutions as discussed in Refs. [20,21.
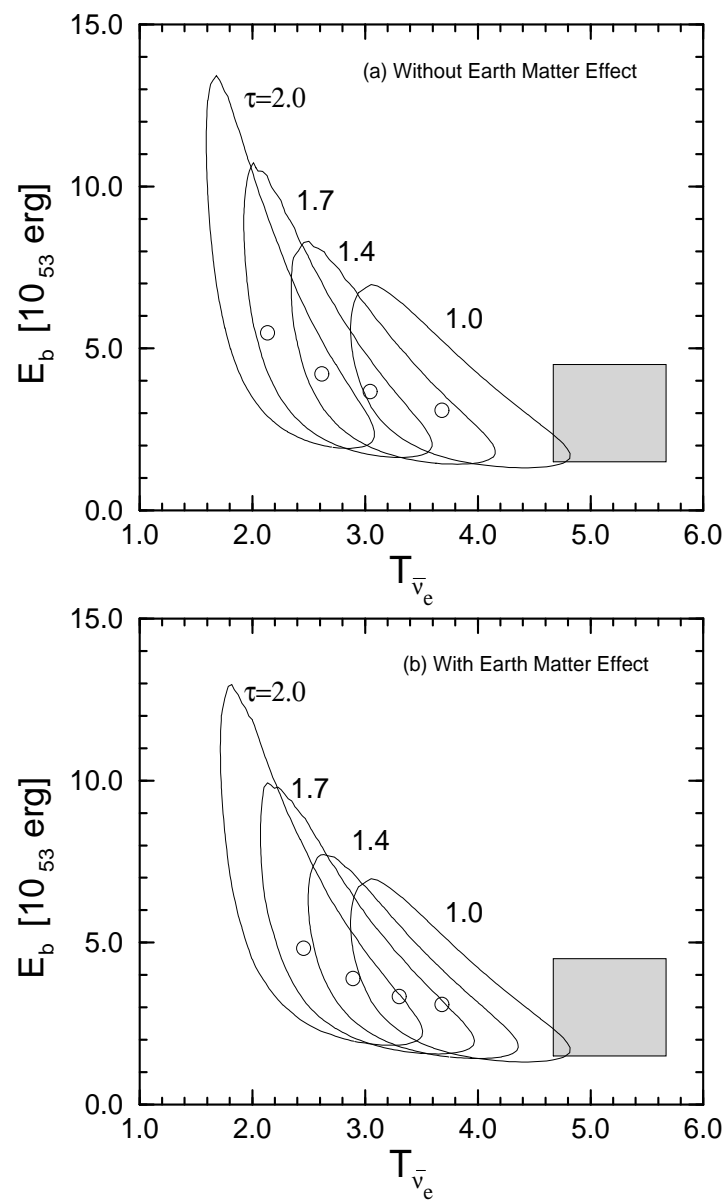

Fig. 5: Contours of constant likelihood corresponding to $95.4 \%$ C.L. for the large mixing angle MSW solution (a) without and (b) with earth matter effect. We have taken mixing parameters as $\Delta m^{2}=3 \times 10^{-5} \mathrm{eV}^{2}$ and $\sin ^{2} 2 \theta=0.8$ for LMA MSW solution. Adopted from Ref. 9

Let us try to demonstrate explicitly this point. We employ a particular set of parameters of the LMA MSW solution and compare the behavior of the likelihood contours with and without earth matter effect. We show our results in Fig. 5. For simplicity, we set $\theta_{13}=0$ but our result does not change much as long as the parameter is under the CHOOZ bound [36]. We see that inconsistency 
between the fitted data and theoretical prediction is somewhat weakened when earth matter effect is included.

\subsection{Case III}

Finally, let us consider the case where mass hierarchy is inverted but $\mathrm{H}$ resonance is moderately adiabatic, which means that $P_{H}$ is not so close to zero or to unity, which can correspond to the case with $\sin ^{2} \theta_{13} \sim 10^{-4}-10^{-5}$.

In this case, $\bar{\nu}_{e}-\bar{\nu}_{\text {heavy }}$ transformation occurs with the probability $1-P_{H}$, and it would imply the similar but milder effect than that we have obtained with a good adiabaticity of the $\mathrm{H}$ resonance, shown in Fig. 4. If the next galactic supernova is detected by Superkamiokande, then we will be able to discriminate the moderately nonadiabatic case from the adiabatic one.

\section{Summary}

We have discussed the possible influence of neutrino oscillation for supernova physics, in particular for observation of $\bar{\nu}_{e}$ spectrum. We stress that the mass spectrum can be tested by $\bar{\nu}_{e}$ signal from supernovae, if $\theta_{13}$ is not very small. We performed some analysis of SN1987A data in the context of three flavor mixing and conclude that inverted mass hierarchy is disfavored by the data unless $\theta_{13}$ is very small, $\sin ^{2} \theta_{13} \lesssim 10^{-4}$. We hope that future galactic supernova may provide more clear information.

\section{ACKNOWLEDGMENTS}

The author thanks H. Minakata for collaboration, A. Yu Smirnov, C. Lunardini, M. C. Gonzalez-Garcia, and R. Tomàs for useful discussions. This work was supported by the Brazilian funding agency Fundação de Amparo à Pesquisa do Estado de São Paulo (FAPESP).

\section{REFERENCES}

1. K. S. Hirata et al., Phys. Rev. Lett. 58 (1987) 1490; Phys. Rev. D38 (1988) 448.

2. R. M. Bionta et al., Phys. Rev. Lett. 58 (1987) 1494.

3. H. Suzuki, in Physics and Astrophysics of
Neutrinos, edited by M. Fukugita and A. Suzuk, Springer Verlag, Tokyo, 1994.

4. G. G. Raffelt, Stars as Laboratories for Fundamental Physics, Univ. of Chicago Press, Chicago, 1996; See also talk by G. Raffelt, in these proceedings.

5. S. P. Mikheyev and A. Yu. Smirnov, Nuovo Cim. 9C (1986) 17; L. Wolfenstein, Phys. Rev. D17 (1978) 2369.

6. L. Wolfenstein, Phys. Rev. D20 (1979) 2634; S. P. Mikheyev and A. Yu. Smirnov, Proceedings of 6th Moriond Workshop on Massive Neutrinos in Astrophysics and Particle Physics, Tignes, France, edited by O. Fackler and J. Tran Thanh Van (Editions Frontieres, Gif-sur-Yvette, 1986) page 355. D. Nötzold, Phys. Lett. B196 (1987) 315; J. Arafune, M. Fukugita, T. Yanagida, and M. Yoshimura, Phys. Rev. Lett. 59 (1987) 1864; H. Minakata, H. Nunokawa, K. Shiraishi, and H. Suzuki, Mod. Phys. Lett. A2 (1987) 827; H. Minakata and H. Nunokawa, Phys. Rev. D38 (1988) 3605; S. P. Rosen, ibid. D37 (1988) 1682; T. K. Kuo and J. Pantaleone, ibid. D37 (1988) 298; T. P. Walker and D. N. Schramm, Phys. Lett. B195 (1987) 331; G. M. Fuller, J. R. Primack, and Y.-Z, Qian, Phys. Rev. D52 (1995) 1288; A. Barrows, D. Klein, and R. Gandhi, ibid. D45 (1992) 3361; G. M. Fuller, W. C. Haxton, and G. C. McLaughlin, ibid. D59 (1999) 085005.

7. G. M. Fuller et al., Astrophys. J 389 (1992) 517.

8. Y.-Z. Qian et al., Phys. Rev. Lett. 71 (1993) 1965.

9. H. Minakata and H. Nunokawa, hep$\mathrm{ph} / 00010240$.

10. R. Meyle, J. R. Wilson, and D. N. Schramm, Astrophys. J. 318 (1987) 288.

11. J. R. Wilson, R. Meyle, S. Woosley, and T. Weaver, Ann. N.Y. Acad. Sci. 470 (1986) 267; A. Barrows and J. M. Lattimer, Astrophys. J. 307 (1986) 178.

12. H.-T. Janka, in Vulcano Workshop 1992; Frontier Objects in Astrophysics and Particle Physics, Proceedings of the Workshop Vulcano, Italy, 1992, edited by F. Giovannelli and G. Mannochi, IPS Conf. Proc. No. 40 (Italian 
Physical Society, Vulcano, 1993).

13. S. W. Bruenn, Phys. Rev. Lett. 59 (1987) 938; A. Barrows, Astrophys. J. 334 (1988) 891; E. S. Myra and A. Barrows, ibid. 364 (1990) 222.

14. H.-T. Janka, Astron. Astrophys. 224 (1989) 49; Astron. Astrophys. Suppl. 78 (1989) 375; P. M. Giovanoni, P. C. Ellison, and S. W. Bruenn, Astrophys. J. 342 (1989) 416.

15. J. R. Wilson, Numerical Astrophysics ed. J. M. Centrella, J. M. Leblanc and R. L. Bowers, p.422 (Boston, Jones and Bartlett, 1983).

16. S. E. Woosley and E. Baron, Astrophys. J. 391 (1992) 228; S. E. Woosley, Astron. Astrophys. Suppl. Ser. 97 (1993) 205; S. E. Woosley and R. D. Hoffman, Astrophys. J. 395 (1992) 202; B. S. Meyer et al., Astrophys. J. 399 (1992) 656; S. E. Woosley et al., Astrophys. J. 433 (1994) 229.

17. H. Minakata and H. Nunokawa, Phys. Rev. D41 (1990) 2976.

18. W. Haxton, Phys. Rev. D36 (1987) 2283.

19. Y.-Z. Qian and G. M. Fuller, Phys. Rev. D49 (1994) 1762.

20. A. Yu. Smirnov, D. N. Spergel, and J.N. Bahcall, Phys. Rev. D49 (1994) 1389.

21. B. Jegerlehner, F. Neubig and G. Raffelt, Phys. Rev. D54 (1996) 1194.

22. D. Cline, astro-ph/0010339.

23. Y. Fukuda et al. (Kamiokande collaboration), Phys. Lett. B335 (1994) 237; Y. Fukuda et al. (SuperKamiokande collaboration), Phys. Rev. Lett. 81 (1998) 1562; T. Kajita, in Neutrino Physics and Astrophysics, Proceedings of the XVIIIth International Conference on Neutrino Physics and Astrophysics (Neutrino '98), June 4-9, 1998, Takayama, Japan, edited by Y. Suzuki and Y. Totsuka, (Elsevier Science B.V., Amsterdam, 1999) page 123.

24. Homestake Collaboration, K. Lande et al., Astrophys .J. 496, 505 (1998); SAGE Collaboration, J. N. Abdurashitov et al., Phys. Rev. C 60, 055801 (1999); GALLEX Collaboration, W. Hampel et al., Phys. Lett. B 447, 127 (1999); Kamiokande Collaboration, Y. Fukuda et al. Phys. Rev. Lett. 77, 1683 (1996); SuperKamiokande Collaboration, Y. Fukuda et al., Phys. Rev. Lett. 81, 1158
(1998); ibid. 81, 4279 (1998); ibid. 82, 2430 (1999); ibid. 82, 1810 (1999).

25. C. Athanassopoulos et al. , for LSND collaboration, Phys. Rev. Lett. 77 (1996) 3082; Phys. Rev. C54 (1996) 2685.

26. D. O. Caldwell, G. M. Fuller and Y-Z. Qian, Phys. Rev. D61 (2000) 123005.

27. O. L. G. Peres and A. Yu. Smirnov, hepph/0011054; See also talk by O. L. G. Peres in these proceedings.

28. H. Murayama and T. Yanagida, hepph/0010178.

29. JHF Neutrino Working Group, Y. Itow et al., Letter of Intent: A Long Baseline Neutrino Oscillation Experiment using the JHF $50 \mathrm{GeV}$ Proton-Synchrotron and the SuperKamiokande Detector, February 3, 2000, http://neutrino.kek.jp/jhfnu

30. The MINOS Collaboration, P. Adamson et al., MINOS Detectors Technical Design Report, Version 1.0, NuMI-L-337, October 1998.

31. OPERA Collaboration, M. Guler et al., OPERA: An Appearance Experiment to Search for $\mathrm{Nu} / \mathrm{Mu} \leftarrow \rightarrow \mathrm{Nu} / \mathrm{Tau}$ Oscillations in the CNGS Beam. Experimental Proposal, CERN-SPSC-2000-028, CERN-SPSC-P-318, LNGS-P25-00, Jul 2000.

32. H. Minakata, Talk at Workshop on Neutrino Oscillations and Their Origin, Fujiyoshida, Japan, February 11-13, 2000, hepph/0004249, to appear in Proceedings; F. Vissani, JHEP 9906 (1999) 022; H.V.KlapdorKleingröthaus, H. Päs and A. Yu. Smirinov, hep-ph/0003219.

33. K. Nomoto and M. Hashimoto, Phys. Rep. 163 (1988) 13. See also the figures in H. Minakata and H. Nunokawa in [6].

34. A. S. Dighe and A. Yu. Smirnov, Phys. Rev. D62 (2000) 033007.

35. C. Lunardini and A. Yu. Smirnov, hepph/0009356; See also the talk by C. Lunardini in these proceedings.

36. CHOOZ Collaboration, M. Apollonio et al., Phys. Lett. B420 (1998) 397; ibid., B466 (1999) 415. See also, The Palo Verde Collaboration, F. Boehm et al., hep-ex/0003022. 